\title{
Reproductive performance and gestational effort in relation to dietary fatty acids in guinea pigs
}

\author{
Matthias Nemeth ${ }^{1 *}$ D, Eva Millesi ${ }^{1}$, Carina Siutz ${ }^{1}$, Karl-Heinz Wagner ${ }^{2}$, Ruth Quint ${ }^{2}$ and Bernard Wallner ${ }^{1,3}$
}

\begin{abstract}
Background: Dietary saturated (SFAs) and polyunsaturated (PUFAs) fatty acids can highly affect reproductive functions by providing additional energy, modulating the biochemical properties of tissues, and hormone secretions. In precocial mammals such as domestic guinea pigs the offspring is born highly developed. Gestation might be the most critical reproductive period in this species and dietary fatty acids may profoundly influence the gestational effort. We therefore determined the hormonal status at conception, the reproductive success, and body mass changes during gestation in guinea pigs maintained on diets high in PUFAs or SFAs, or a control diet.

Results: The diets significantly affected the females' plasma fatty acid status at conception, while cortisol and estrogen levels did not differ among groups. SFA females exhibited a significantly lower body mass and litter size, while the individual birth mass of pups did not differ among groups and a general higher pup mortality rate in larger litters was diminished by PUFAs and SFAs. The gestational effort, determined by a mother's body mass gain during gestation, increased with total litter mass, whereas this increase was lowest in SFA and highest in PUFA individuals. The mother's body mass after parturition did not differ among groups and was positively affected by the total litter mass in PUFA females.
\end{abstract}

Conclusions: While SFAs reduce the litter size, but also the gestational effort as a consequence, PUFA supplementation may contribute to an adjustment of energy accumulations to the total litter mass, which may both favor a mother's body condition at parturition and perhaps increase the offspring survival at birth.

Keywords: Body mass, Female reproduction, Gestation, Litter size, Polyunsaturated fatty acid, Saturated fatty acid, Total litter mass

\section{Background}

Reproduction represents the energetically most demanding life history stage in mammalian females. Adequate and balanced dietary intakes of specific macronutrients are of major importance to ensure an appropriate energy supply for maintaining reproductive performances [1]. In this context, dietary fatty acids have been suggested to play a major role in providing energy for an organism as well as by affecting hormone secretions and cell membrane functions in the central nervous system and the reproductive tract. The dietary fatty acid content and composition, particularly the amounts and ratios of

\footnotetext{
*Correspondence: matthias.nemeth@univie.ac.at

'Department of Behavioural Biology, University of Vienna, Althanstrasse 14,

1090 Vienna, Austria

Full list of author information is available at the end of the article
}

specific types of fatty acids in the diet, can therefore directly modulate the physiology of reproduction and ultimately an individual's reproductive success $[2,3]$.

Among dietary fat types, omega-3 (n-3) and omega- 6 ( $\mathrm{n}-6)$ polyunsaturated fatty acids (PUFAs), including the essential $\alpha$-linolenic acid (ALA, 18:3 n-3) and linoleic acid (LA, 18:2 n-6) and their long-chain metabolites eicosapentaenoic acid (EPA, 20:5 n-3), docosahexaenoic acid (DHA, 22:6 n-3), and arachidonic acid (AA, 20:4 $\mathrm{n}-6)$, can positively influence reproductive processes in females. Diets high in ALA or in the n-3 long-chain metabolites EPA and DHA have been shown to promote ovulation and increase the number of released ova in rats [4], and may further increase conception rates and reduce pregnancy losses in cows [5]. PUFAs in general, including ALA and LA, can also promote the prenatal 
development of rabbits and mice, resulting in increased body mass and improved physical condition at birth $[6,7]$. Although less well studied, dietary intakes of non-essential saturated fatty acids (SFAs) can also improve reproductive performances by increasing the birth mass in rats [8] or the sex ratio at birth towards more male offspring in mice [9]. However, dietary SFAs may not only promote reproductive functions as PUFAs obviously do, since various effects on metabolic processes may be detrimental for an individual $[10,11]$. The effects of dietary fatty acids may be simply explained by their high energy content and therefore increased energy allocation for reproductive functions and prenatal development, or by improving a mother's body condition and hormone secretion rates already at the time of conception [3].

By modulating litter size, sex ratio, and the offspring's birth mass, dietary fatty acids not only affect the reproductive output, but also a female's body mass change during pregnancy, reflecting the gestational effort. Especially in precocial mammalian species such as the domestic guinea pig (Cavia aperea f. porcellus) pups are born highly developed and gestation is basically characterized by a very high investment of mothers [12]. Larger litter sizes obviously cause higher gestational efforts in guinea pigs and are usually characterized by a lower birth mass of single pups and a higher rate of stillbirths compared to smaller litters $[13,14]$. Although lactation in guinea pigs definitely represents an important and energetically demanding period, this may be less pronounced compared to altricial mammalian species. The prolonged gestation period can be assumed to be the energetically more demanding reproductive period in guinea pigs, especially as newborn pups are of relatively high body mass and the lactation period occurs to be rather short $[15,16]$. Since mortality in term-born guinea pig pups is relatively low [17], probably due to the high developmental stage at birth, reproduction in guinea pigs can be considered as highly efficient and could probably be further promoted by supplementations with dietary fatty acids.

The aim of this study was therefore to determine and compare the effects of diets high in PUFAs or SFAs on reproductive output, offspring survival and condition, and body mass changes in female guinea pigs during gestation. Due to the relatively long gestation period and the precociality, guinea pigs may represent an adequate model species to study the effects of dietary fatty acids during gestation and prenatal development. Knowledge on such influences may further increase the understanding for the relevance of dietary fatty acids regarding an individual's reproductive success in general, but also how these nutrients may influence the process of gestation in mothers in relation to the offspring's prenatal development.

\section{Methods}

\section{Ethical statement}

Experiments were conducted in accordance with EU Directive 2010/63/EU for animal experiments and the Austrian laws for animal experiments and animal keeping. The study has been checked and approved by the internal board on animal ethics and experimentation of the Faculty of Life Sciences of the University of Vienna (\# 2014-005) and the Austrian Federal Ministry of Science and Research (BMWF-66.006/0024-II/3b/2013).

\section{Animals and housing conditions}

All domestic guinea pigs ( 30 males and 30 females) used for this study were bred at the Department of Behavioural Biology at the University of Vienna. All animals were adult, sexually intact, and accustomed to the daily contact with humans. Differences in natural fur colorations allowed an individual identification. Animals were housed in single-sexed groups of ten individuals, resulting in three male and three female groups. Each group's enclosure $(2 \mathrm{~m} \times 1.6 \mathrm{~m})$ was environmentally enriched with shelters and platforms and the floor was covered with standard bedding material. Animals were housed at a temperature of $20 \pm 2{ }^{\circ} \mathrm{C}, 50 \pm 5 \%$ humidity, and a light-dark cycle of $12 \mathrm{~h}$ with lights on at $0700 \mathrm{~h}$.

The daily provided food consisted of guinea pig pellets (ssniff V2233, ssniff Spezialdiäten GmbH, Soest, Germany) and $50 \mathrm{~g}$ of hay per group; for precise nutrient composition of guinea pig pellets see [18]. According to the manufacturer's information, guinea pig pellets contain 3\% (w/w) crude fat, which accounts for $8 \%$ of the convertible energy provided with the food (gross energy content of guinea pig pellets: $16.6 \mathrm{MJ} / \mathrm{kg}$; see Table 1 for fatty acid composition of the guinea pig pellets). Water was provided in several drinking bottles. In addition to the daily provided standard food, each male and female group received one of three different dietary supplements: walnut oil (high in PUFAs; gross energy content: $38.7 \mathrm{MJ} / \mathrm{kg}$, fat content: 99.6\%), coconut fat (high in SFAs; gross energy content: $38.9 \mathrm{MJ} / \mathrm{kg}$, fat content: $100 \%$ ), or pure water in case of a control group (see Table 1 for fatty acid compositions of walnut oil and coconut fat). Each animal was administered orally with $3 \mathrm{~mL}$ of the specific supplement per $1 \mathrm{~kg}$ body mass on every day of the experiment using $1 \mathrm{~mL}$ syringes. This procedure, including the supplemented amounts, has already been applied previously in studies on the effects of dietary fatty acids in rats and guinea pigs and proved successful in affecting the fatty acid status of the individuals $[19,20]$ and result approximately in $25 \%$ energy intake from dietary fats in these animals. An imbalance in the total energy intake among single individuals and the three dietary groups, which would have been caused by the additional fatty acid supplementations, was counteracted by ad libitum feeding of guinea pig pellets. 
Table 1 Most prominent fatty acids (\% of total fatty acids based on gas chromatography analyses) of guinea pig pellets, walnut oil, and coconut fat

\begin{tabular}{llll}
\hline Fatty acid & Guinea pig pellets $^{a}$ & Walnut oil & Coconut fat \\
\hline C 12:0 & n.d. & n.d. & 49.26 \\
C 14:0 & 0.61 & n.d. & 22.53 \\
C 16:0 & 16.16 & 6.55 & 11.82 \\
C 16:1 & 0.61 & n.d. & n.d. \\
C 18:0 & 3.35 & 2.76 & 4.60 \\
C 18:1 n-9 & 18.90 & 14.16 & 6.71 \\
C 18:1 n-7 & n.d. & 1.28 & 0.53 \\
C 18:2 n-6 & 50.00 & 63.14 & 3.73 \\
C 18:3 n-6 & n.d. & 0.40 & 0.26 \\
C 18:3 n-3 & 10.06 & 11.03 & n.d. \\
total MUFAs & 19.51 & 15.44 & 7.39 \\
total PUFAs & 60.06 & 75.25 & 4.22 \\
total SFAs & 20.43 & 9.31 & 88.39 \\
P:S ratio & 2.94 & 8.08 & 0.05 \\
\hline
\end{tabular}

n.d. Not detectable

${ }^{a}$ Ssniff V2233, ssniff Spezialdiäten GmbH, Soest, Germany

Guinea pig pellets were provided ad libitum in each dietary group; walnut oil (PUFA group) and coconut fat (SFA group) were additionally supplemented

( $3 \mathrm{~mL} / \mathrm{kg}$ body mass) to animals of the corresponding group

\section{Experimental procedure}

Animals of the different groups were compared in their age and body mass in advance to the experiment to exclude possible differences in these variables at the onset of the study. Males were included in these preexperimental analyses in order to exclude any possible influences of male body condition on female reproductive performance. Using the statistical package $\mathrm{R}$ 3.2.2 [21] and two-way analyses of variance, no differences among the dietary and/or sex groups were found in age $\left(F_{5,54}=0.566, P=0.726\right.$; mean age: $21.1 \pm 1.2$ month $)$ or body mass $\left(F_{5,54}=0.746, P=0.593\right.$; mean body mass: $805 \pm 20 \mathrm{~g})$ at the beginning of the experiment.

The experimental procedure started with an initial 100-d feeding phase. All animals were weighed daily at $0900 \mathrm{~h}$ and the body mass-based supplementations were carried out, which lasted no longer than $1 \mathrm{~min}$ in total per animal. The dietary supplementations for $100 \mathrm{~d}$ should ensure that the individuals had highest possible levels of specific fatty acids and a maximum incorporation into neuronal membranes and tissues of the reproductive tracts. After d 100, blood samples were collected from all animals to measure plasma fatty acid levels as indicators of the general fatty acid status [22, 23] and females were mated with heterogeneous males maintained on the same dietary supplements as mentioned above. For this purpose, male and female groups, supplemented with the same fatty acids, were randomly mixed in two mating groups, each consisting of five males and five females. This yielded a total of six mating groups, two for each dietary regime. Mating in small groups with a sex ratio of 1:1 should reduce male competition for females and increase the number of social partners in order to reduce social stress [24] and, therefore, reliably result in frequent pregnancy rates. During the mating period, saliva and plasma samples were collected from females to analyze saliva cortisol and plasma estrogen levels at conception. Both hormones served as indicators of female homeostasis at conception. Once pregnancy was detected, the respective female was removed from the mating group and introduced to its single-sexed group again. Weighing and dietary supplementation procedures continued throughout the experiment and were still carried out daily at $0900 \mathrm{~h}$. until the first day after parturition.

\section{Measurements of gestational and reproductive performance}

During mating, the vaginal membrane of each female was inspected visually to monitor receptivity during the estrous cycle, because the vagina appears to be opened at this stage of proestrus/estrus for 1-6 d [25]. To define the day of conception, a time span of approximately 66-69 d was counted back from the day of parturition. This constant period of gestation is well documented in guinea pigs (see for example [26]). The first day with an opened vagina within this time frame represented the day of conception. Due to a relatively long estrous cycle in guinea pigs of about $16 \mathrm{~d}$ [27], a misinterpretation was very unlikely, as this would have resulted in unnaturally short $(\sim 50 \mathrm{~d})$ or long $(\sim 85$ d) gestation periods. The number of days from conception to parturition was defined as gestation duration.

Most females gave birth during the night and therefore the offspring of each female was counted, sexed, and weighed $12 \mathrm{~h}$ after parturition at the latest. Also pups that were found dead, but fully developed, were weighed and sexed. The body mass of mothers at conception, during gestation, on the last day of gestation (1 d before parturition), and after parturition was recorded using a standard electronic scale (accuracy \pm 1 g) to monitor changes in body mass throughout gestation and parturition.

\section{Saliva and blood sampling procedures}

Saliva samples were collected by inserting standard cotton buds into the animal's mouth for approximately $1 \mathrm{~min}$. Cotton buds were then centrifuged $(14,000 \mathrm{rpm}$, $10 \mathrm{~min}$ ) and pure saliva was stored at $-20{ }^{\circ} \mathrm{C}$ until further analysis. Blood was collected with heparinized micropipettes after punctuation of prominent ear veins. Plasma was separated by centrifugation $(14,000 \mathrm{rpm}$, $10 \mathrm{~min}$ ) and stored at $-20{ }^{\circ} \mathrm{C}$ until further analysis (for further information regarding sample collection procedures see [28]). 


\section{Hormone analyses}

Hormone concentrations in saliva and plasma were analyzed by biotin-strepdavidin enzyme-linked immunoassays [29, 30]. Saliva samples were diluted 1:50 and cortisol concentrations measured in $10 \mu \mathrm{L}$ aliquots using a cortisol-specific antibody. Extraction of plasma hormones was done by adding $2 \mathrm{~mL}$ diethylether to $100 \mu \mathrm{L}$ plasma, shaking the samples four times for $15 \mathrm{~min}$, and freezing them overnight. After evaporation of the diethylether $\left(30{ }^{\circ} \mathrm{C}, 10 \mathrm{~min}\right)$, samples were diluted $1: 4$ and concentrations of total estrogens in plasma were determined in $25 \mu \mathrm{L}$ aliquots using an antibody against total estrogens. For further information regarding the used antibodies, including cross- reactions with relevant steroids, see Palme \& Möstl [29, 30]. All analyses were run in duplicates. The confidence criterion was set at $\leq 15 \%$ for the coefficient of variance of the sample duplicates. Intraassay coefficients of variance for saliva cortisol and plasma estrogen concentrations were 11.15 and 8.64\%, respectively.

\section{Plasma fatty acid analyses}

Proportions of fatty acids in plasma prior to mating and the gestational period were analyzed by gas chromatography. Following the protocols by Wagner et al. [31] and Nemeth et al. [22], fatty acids in $35 \mu \mathrm{L}$ plasma were transesterificated by adding $1 \mathrm{~mL}$ methanolic $\mathrm{NaOH}$, containing butylated hydroxytoluene, and $1 \mathrm{~mL}$ borontrifluoride to obtain fatty acid methyl esters (FAMES). Samples were boiled for $5 \mathrm{~min}$ at $100{ }^{\circ} \mathrm{C}$ and cooled on ice for $10 \mathrm{~min}$ after each step. FAMES were then extracted by adding $500 \mu \mathrm{L}$ hexane four times, including 5 min of shaking the samples $(700 \mathrm{rpm})$ in between each addition. Samples were evaporated at $40{ }^{\circ} \mathrm{C}$ under nitrogen and redissolved in hexane. FAMES were separated by an Auto-System-Gaschromatograph (Perkin Elmer, USA) with flame ionization detector, equipped with an Rtx-2330 $30 \mathrm{~m} \times 0.25 \mathrm{~mm} \times 0.20 \mu \mathrm{m}$ silica column. $1 \mu \mathrm{L}$ of prepared samples was injected under a 1:25 split at $250{ }^{\circ} \mathrm{C}$ and detected at $275^{\circ} \mathrm{C}$; helium was used as carrier gas. Fatty acids were identified using a 37 component FAME Mix Standard (Supelco, Bellafonte, USA) and TotalChrome Workstation 6.3.0 (PE Nelson, Perkin Elmer, USA) was used for peak integration. Fatty acids are expressed as percentage of total fatty acids.

\section{Statistical analyses}

Statistical analyses were carried out using $\mathrm{R}$ version 3.2.2 [21] and the implemented package 'nlme' [32] for performing linear mixed effect models (LMEs). For post-hoc analyses, the packages 'phia' [33] and 'PMCMR' [34] were used. Package 'effects' [35] was used to extract effect plots.

Based on the distribution of the data, conditions at conception and reproductive parameters were analyzed and compared among groups (control, PUFA, SFA) using one-way analyses of variance (ANOVAs), KruskalWallis, or Pearson's Chi-squared tests. The sex ratio and survival rate at birth were analyzed by generalized linear models (GLMs) with binomial link. Single pup's birth mass was analyzed using LMEs, including 'group', 'sex', and their interaction as fixed effects, and 'mother' as random effect to correct for the relatedness. To control for possible litter size effects, 'litter size' was included in the models as covariate.

The body mass during gestation was analyzed by an LME, including 'group' (control, PUFA, SFA), 'day' as second-order polynomial term, and 'total litter mass', as well as their interactions as fixed effects, and individual ID' as random effect to correct for repeated measurements. The second-order polynomial of 'day' was also included as random slope to correct for individual changes in body mass during gestation. In the beginning, models including either 'total litter mass' or 'litter size' as covariate were compared based on the Akaike information criterion (AIC). This analysis suggested to include total litter mass rather than litter size as fixed effect, although litter size is usually used as covariate to analyze the body mass during gestation (see for example [26]). Total litter mass was also preferred due to the relatively low variation of litter size in the SFA group and should definitely represent the litter size effect, as litter size and total litter mass were highly related $\left(R^{2}=0.87, P=3 \times 10^{-13}\right)$.

To analyze the gestational effort, body mass in females prior to parturition and on the first day after parturition were first compared among groups by ANOVAs. Afterwards, linear models were calculated, including 'group' (control, PUFA, SFA), 'body mass at conception', and 'total litter mass', as well as their interactions as predictor variables, therefore reflecting the gestational effort as the body mass increase during gestation corrected for the body mass at conception.

Model assumptions (linearity, normality, homoscedasticity of residuals) were checked by performing ShapiroWilk normality tests and Levene's test for homogeneity of variance as well as by model diagnostic plots of residuals and fitted values. Models were fitted (removal of non-relevant interaction and main effects) based on the AIC. Only the highest significant interaction and/or main effects are considered in the result section. Model statistics are based on type 3 sum of squares. All posthoc analyses were Bonferroni corrected. The level of significance was set at $P \leq 0.05$.

\section{Results}

Plasma fatty acids

The dietary treatments resulted in different plasma fatty acid proportions among the female groups (Table 2). According to the diets, SFA females showed highest 
Table 2 Most prominent plasma fatty acids (\% of total plasma fatty acids) in female guinea pigs maintained on a control, high-PUFA, or high-SFA diet

\begin{tabular}{|c|c|c|c|c|c|}
\hline Fatty acid & Control & PUFA & SFA & $F$-value & $P$-value \\
\hline C 12:0 & $0.06 \pm 0.02^{\mathrm{a}}$ & $0.07 \pm 0.02^{a}$ & $1.27 \pm 0.36^{b}$ & 30.059 & $<0.001$ \\
\hline C 14:0 & $0.93 \pm 0.08^{\mathrm{a}}$ & $0.59 \pm 0.04^{a}$ & $4.64 \pm 0.61^{b}$ & 88.240 & $<0.001$ \\
\hline C 16:0 & $17.12 \pm 0.23^{a}$ & $12.70 \pm 0.39^{b}$ & $16.80 \pm 0.49^{a}$ & 40.270 & $<0.001$ \\
\hline C $18: 0$ & $10.09 \pm 0.52^{\mathrm{a}}$ & $6.84 \pm 0.41^{b}$ & $9.05 \pm 0.40^{a}$ & 113.780 & $<0.001$ \\
\hline C 18.1 n9 & $15.53 \pm 0.46^{a}$ & $12.85 \pm 0.70^{b}$ & $12.49 \pm 0.59^{b}$ & 7.901 & 0.002 \\
\hline C 18:2 n6 & $39.77 \pm 1.00^{\mathrm{a}}$ & $49.13 \pm 0.86^{b}$ & $41.73 \pm 0.60^{a}$ & 34.695 & $<0.001$ \\
\hline C $18: 3$ n3 & $6.02 \pm 0.22^{a}$ & $7.71 \pm 0.14^{b}$ & $4.47 \pm 0.36^{c}$ & 19.890 & $<0.001$ \\
\hline total n-9 & $16.21 \pm 0.47^{\mathrm{a}}$ & $15.42 \pm 0.46^{a}$ & $13.13 \pm 0.58^{b}$ & 9.991 & $<0.001$ \\
\hline total n-6 & $43.09 \pm 0.93^{\mathrm{a}}$ & $51.41 \pm 0.90^{b}$ & $44.62 \pm 0.67^{\mathrm{a}}$ & 27.577 & $<0.001$ \\
\hline total n-3 & $6.64 \pm 0.24^{a}$ & $8.12 \pm 0.45^{b}$ & $4.95 \pm 0.36^{c}$ & 19.400 & $<0.001$ \\
\hline total SFA & $31.67 \pm 0.74^{a}$ & $23.74 \pm 0.91^{b}$ & $35.31 \pm 0.81^{c}$ & 51.931 & $<0.001$ \\
\hline total MUFA & $18.51 \pm 0.50^{a}$ & $16.66 \pm 0.49^{b}$ & $15.03 \pm 0.63^{b}$ & 10.111 & $<0.001$ \\
\hline total PUFA & $49.82 \pm 1.08^{\mathrm{a}}$ & $59.60 \pm 0.89^{b}$ & $49.66 \pm 0.55^{a}$ & 43.030 & $<0.001$ \\
\hline n-6: n-3 ratio & $6.55 \pm 0.22^{a}$ & $6.57 \pm 0.51^{a}$ & $9.59 \pm 0.88^{b}$ & 9.254 & $<0.001$ \\
\hline$P$ : S ratio & $1.59 \pm 0.07^{a}$ & $2.56 \pm 0.13^{b}$ & $1.42 \pm 0.04^{a}$ & 50.46 & $<0.001$ \\
\hline
\end{tabular}

Different superscript letters indicate significant differences between single groups $(P \leq 0.05)$

percentages in total SFAs, while PUFA females showed lowest percentages in all types of SFAs. PUFA females, in contrast, showed highest percentages in $n-3$ and $n-6$ PUFAs, while n-9 MUFAs were highest in control females. The plasma n-6:n-3 ratio was significantly higher in SFA females, while PUFA females showed the highest $\mathrm{P}: \mathrm{S}$ ratio.

\section{Body conditions at conception}

The body mass at conception, approximately $100 \mathrm{~d}$ after the onset of the feeding procedure, differed among the groups $\left(F_{2,25}=6.954, P=0.004\right)$ and was significantly lower in SFA females compared to control females (control: $942 \pm 22$ g; PUFA: $938 \pm 50$ g; SFA: $841 \pm 15$ g; control-PUFA: $\chi^{2}=0.006, P=1.000$; control-SFA: $\chi^{2}=$ 13.998, $P=0.001$; PUFA-SFA: $\left.\chi^{2}=3.447, P=0.190\right)$. No differences were detected in saliva cortisol concentrations $\left(F_{2,25}=1.891, P=0.172\right.$; control: $18.77 \pm 4.45 \mathrm{ng} / \mathrm{mL}$; PUFA: $58.97 \pm 23.81 \mathrm{ng} / \mathrm{mL}$; SFA: $40.24 \pm 12.68 \mathrm{ng} / \mathrm{mL}$ ) or plasma estrogen levels $\left(F_{2,17}=2.472, P=0.114\right.$; control: $0.48 \pm 0.09 \mathrm{ng} / \mathrm{mL}$; PUFA: $0.39 \pm 0.08 \mathrm{ng} / \mathrm{mL}$; SFA: $0.24 \pm$ $0.07 \mathrm{ng} / \mathrm{mL}$ ) at conception.

\section{Overall reproductive output}

A total of 28 female guinea pigs became pregnant and showed successful gestations, resulting in normal parturitions at term. Most females became pregnant in their first estrus during the mating phase, with no differences among groups in the number of estrous cycles until conception $\left(\chi^{2}=2.505, P=0.286\right)$. A total of 85 pups were born fully developed (52 $\hat{\sigma}: 33$ ㅇ). Except for a total litter loss of five pups at birth in one control female, the number of fully-developed, but dead-born pups was very low (71 alive : 14 dead). All dead-born pups were found in their amniotic sacs. The overall reproductive output, including pregnancy rates, total number of pups, total sex ratio, and ratio of alive and dead born pups, did not differ among the groups (Table 3).

Table 3 Statistical analysis of the overall reproductive output for groups of female guinea pigs ( $n=10$ per group) maintained on a control, high-PUFA, or high-SFA diet

\begin{tabular}{|c|c|c|c|c|c|}
\hline Reproductive parameter & Control & PUFA & SFA & $x^{2}$ & $P$-value \\
\hline Number of pregnancies & 10 & 8 & 10 & 0.286 & 0.867 \\
\hline Total offspring number & 33 & 28 & 24 & 1.435 & 0.488 \\
\hline Total sex ratio & 19 ठૈ: 14 ㅇ & 18 ㅈ: 10 우 & 150 : 9 q & 0.312 & 0.856 \\
\hline Alive : Dead ratio & $24 \mathrm{~A}: 9 \mathrm{D}$ & $25 \mathrm{~A}: 3 \mathrm{D}$ & $22 \mathrm{~A}: 2 \mathrm{D}$ & 4.628 & 0.099 \\
\hline Alive : Dead ratio $\sigma^{\pi}$ & $14 \mathrm{~A}: 5 \mathrm{D}$ & $16 \mathrm{~A}: 2 \mathrm{D}$ & $13 \mathrm{~A}: 2 \mathrm{D}$ & 1.726 & 0.422 \\
\hline Alive : Dead ratio $q$ & $10 \mathrm{~A}: 4 \mathrm{D}$ & $9 \mathrm{~A}: 1 \mathrm{D}$ & $9 \mathrm{~A}: 0 \mathrm{D}$ & 3.775 & 0.152 \\
\hline
\end{tabular}




\section{Reproductive parameters}

A significant difference among groups was detected in the litter size $\left(\chi^{2}=6.855, P=0.032\right)$ and the total litter mass $\left(F_{2,25}=4.032, P=0.030\right)$, while the sex ratio per litter $\left(\chi^{2}=\right.$ $0.311, P=0.856)$, the gestation duration $\left(\chi^{2}=0.573, P=\right.$ 0.751 ), the individual birth mass (corrected for the pups' relatedness: $F_{2,25}=1.568, P=0.228$ ), and the survival rate at birth $\left(\chi^{2}=4.548, P=0.103\right)$ did not differ (Table 4). A higher litter size was found in PUFA females compared to SFA females and, correspondingly, a lower total litter mass in the SFA group compared to the control group. Although single pup's birth mass did not differ among groups, males were barely heavier at birth than females $\left(F_{1,56}=\right.$ 4.059, $P=0.049$; males: $110.5 \pm 2.6 \mathrm{~g}$; females: $105.9 \pm 2.8 \mathrm{~g})$.

Including litter size as covariate in the respective analyses revealed that neither the sex ratio $\left(\chi^{2}=0.080, P=0.777\right)$ nor the gestation duration $\left(F_{1,25}=0.871, P=0.360\right)$ was affected by litter size at all. However, the litter size positively affected the total litter mass $\left(F_{1,24}=145.113, P<\right.$ 0.001 ) and had a negative effect on the survival rate at birth $\left(\chi^{2}=7.706, P=0.006\right)$, while only a negative tendency was detected regarding the individual birth mass $\left(F_{1,26}=3.701\right.$, $P=0.065$ ) (Table 5). Therefore, pups born in larger litters were of lower birth mass and faced a higher mortality rate at birth. Although all these influences did not differ among groups (for all reproductive parameters: $P>0.240$ ), the negative effect of litter size on the individual birth mass was only significant in the SFA group, while a significant negative effect of litter size on the survival rate was only detected in the control group (Table 5).

\section{Body mass changes in mothers}

From conception until d 65 of gestation (prior to parturition) the body mass increase in mothers differed significantly among groups (group: $F_{2,22}=0.992, P=0.387$; day: $F_{2,379}=0.611, P=0.544$; total litter mass: $F_{1,22}=2.774$,

Table 4 Reproductive performance of female guinea pigs maintained on a control $(n=10)$, high-PUFA $(n=8)$, or highSFA $(n=10)$ diet

\begin{tabular}{llll}
\hline $\begin{array}{l}\text { Reproductive } \\
\text { parameter }\end{array}$ & Control & PUFA & SFA \\
\hline Litter size $^{1}$ & $3(2-5)^{\mathrm{ab}}$ & $3.5(2-5)^{\mathrm{a}}$ & $3(1-3)^{\mathrm{b}}$ \\
Sex ratio $^{2}$ & $0.56(0.36,0.76)$ & $0.66(0.44,0.89)$ & $0.68(0.49,0.88)$ \\
Gestation duration $^{1}$ & $69(67-70)$ & $68(64-69)$ & $68(67-73)$ \\
Total litter mass $^{3}$ & $368.8 \pm 29.1^{\mathrm{a}}$ & $355.6 \pm 32.5^{\mathrm{ab}}$ & $260.6 \pm 29.1^{\mathrm{b}}$ \\
Individual birth $^{3}$ & $112.1 \pm 3.8$ & $102.3 \pm 4.2$ & $110.5 \pm 4.0$ \\
mass $^{3}$ & & & \\
Survival rate $^{2}$ & $0.73(0.47,0.89)$ & $0.89(0.60,0.98)$ & $0.92(0.58,0.99)$
\end{tabular}

${ }^{1}$ Data given as median and range; analysis via Kruskal-Wallis test

${ }^{2}$ Data given as (mean) effect size and lower and upper confidence limits; analysis via GLM with binomial link

${ }^{3}$ Data given as mean \pm s.e.m; analysis via ANOVA or LME

Different superscript letters indicate significant differences between single groups $(P \leq 0.05)$
$P=0.110$; group:day: $F_{4,379}=3.296, P=0.011$; group:total litter mass: $F_{2,22}=2.169, P=0.138$; day:total litter mass: $F_{2,379}=28.845, P<0.001$; group:day:total litter mass: $F_{4,379}$ $=3.337, P=0.011$ ). The body mass increase in 5 -d intervals was generally less pronounced in SFA females compared to control and PUFA females (control-PUFA day ${ }^{1}: t=1.715, P$ $=0.087$; day $^{2}: t=0.567, P=0.571$; control-SFA day ${ }^{1}: t=$ 1.975, $P=0.049$; day ${ }^{2}: t=0.349, P=0.728$; PUFA-SFA day ${ }^{1}$ : $t=3.433, P=0.001$; day ${ }^{2}: t=0.862, P=0.389$ ) (Fig. 1 ). Statistical modeling and effect plotting revealed that PUFA females showed the strongest increase in body mass as total litter mass increased, whereas this effect was least pronounced in SFA females (Fig. 2).

On the last day of gestation, before parturition, body mass still differed among groups $\left(F_{2,25}=5.759, \quad P=\right.$ 0.009), with SFA females exhibiting a significantly lower body mass compared to the other groups (control: 1441 \pm 52 g; PUFA: $1432 \pm 82$ g; SFA: $1216 \pm 26$ g; controlPUFA: $\chi^{2}=0.013, P=1.000$; control-SFA: $\chi^{2}=9.276, P=$ 0.016; PUFA-SFA: $X^{2}=7.599, P=0.032$ ) (Fig. 1). Corrected for the body mass at conception, the effect of total litter mass on the mother's body mass prior to parturition differed among groups (body mass at conception: $F_{1,21}=38.006, P<0.001$; group: $F_{2,21}=3.579$, $P=0.046$; total litter mass: $F_{1,21}=39.070, \quad P<0.001$; group:total litter mass: $F_{2,21}=3.990, P=0.034$ ) and was more pronounced in PUFA females compared to SFA females (control-PUFA: $F=2.932, P=0.305$; control-SFA: $F=1.864, \quad P=0.560 ; \quad$ PUFA-SFA: $\quad F=7.956, \quad P=0.030)$ (Fig. 3a). The effect of mother's body mass at conception did not differ among groups and was removed from the model beforehand (group:body mass at conception: $\left.F_{2,19}=0.258, P=0.776\right)$.

On the first day after parturition, no differences in body mass were detected among the three groups $\left(F_{2,25}\right.$ $=2.134, P=0.140$ ) (Fig. 1). However, compared to the body mass at conception, all groups were significantly heavier after parturition (control: $+44.7 \mathrm{~g}, t=2.405, p=$ 0.024; PUFA: $+52.38 \mathrm{~g}, t=2.521, P=0.018$; SFA: $+62.8 \mathrm{~g}$; $t=3.379, P=0.002)$. This increase was similar for all groups $\left(F_{2,25}=0.239, P=0.789\right)$. Further analyses revealed that the body mass after parturition, corrected for the body mass at conception, tended to be differently affected by the total litter mass (body mass at conception: $F_{1,21}=48.454, P<0.001$; group: $F_{2,21}=3.118, P=$ 0.065 ; total litter mass: $F_{1,21}=0.445 ; P=0.512$; group:total litter mass: $\left.F_{2,21}=3.167, P=0.063\right)$. While a positive effect of total litter mass on the body mass after parturition was detected for PUFA females $(F=4.708, P=$ 0.042), no effects were detected for control and SFA females (control: $F=0.445, P=0.512$; SFA: $F=1.915$, $P=0.181$ ) (Fig. 3b).

The total body mass loss after parturition was not identical to the total litter mass and therefore the 
Table 5 Effect of litter size on the reproductive performance of female guinea pigs maintained on a control $(n=10)$, high-PUFA $(n=8)$, or high-SFA $(n=10)$ diet and the general effect of litter size

\begin{tabular}{lllll}
\hline Reproductive parameter & Control & PUFA & SFA & Litter size $^{\text {a }}$ \\
\hline Sex ratio $^{b}$ & $0.081(-0.192,0.356)$ & $-0.195(-0.638,-0.055)$ & $-0.042(-0.398,0.170)$ & $-0.017(-0.016,-0.020)$ \\
Gestation duration $^{c}$ & $0.278 \pm 0.675$ & $-0.500 \pm 0.954$ & $-0.281 \pm 0.939$ & $-0.294 \pm 0.315$ \\
Total litter mass $^{c}$ & $110.590 \pm 14.616^{* * *}$ & $90.917 \pm 20.756^{* * *}$ & $95.406 \pm 20.426^{* * *}$ & $98.957 \pm 8.215^{* * *}$ \\
Individual birth mass $^{c}$ & $-2.124 \pm 4.669$ & $-4.144 \pm 6.664$ & $-10.351 \pm 6.950^{*}$ & $-4.929 \pm 2.562$ \\
Survival rate $^{b}$ & $-0.174(-0.382,-0.034)^{*}$ & $-0.035(-0.460,0.036)$ & $-0.056(-0.803,-0.028)$ & $-0.120(-0.020,-0.273)^{*}$ \\
\hline
\end{tabular}

${ }^{*} p \leq 0.05,{ }^{* *} p \leq 0.01,{ }^{* * *} p \leq 0.001$ for significant effects of litter size

${ }^{a}$ Effect of litter size determined after removal of non-significant effects based on the AIC. No significant differences between single groups (for all pairwise comparisons $P>0.05$ )

${ }^{b}$ Data given as (mean) effect size and lower and upper confidence limits; analysis via GLM with binomial link

'Data given as slope \pm standard error of the slope; analysis via linear model or LME

difference probably represented placental tissues and amniotic fluid. This part of the females' body mass loss differed significantly among the groups $\left(F_{2,25}=3.444, P\right.$ $=0.048$ ) and tended to be lower in SFA females (control: $85 \pm 10$ g; PUFA: $86 \pm 14$ g; SFA: $52 \pm 9$ g; post-hoc analyses with Bonferroni corrections remained nonsignificant). This, however, was positively affected by the total litter mass, which also diminished the group difference (group: $F_{2,22}=0.914, P=0.416$; total litter mass: $F_{1,26}=33.442, P<0.001$; group:total litter mass: $F_{2,22}=$ $1.258, P=0.304)$.

\section{Discussion}

A mother's dietary fatty acid intake can profoundly affect the reproductive success and may further determine the post-natal development of her offspring. These effects are suggested to be caused by physiological influences of these nutrients already prior to conception, but even by modulating the processes of gestation and parturition [36-38]. Here we could show that diets high in PUFAs and SFAs differently affect reproductive performances in female guinea pigs. These influences, however, were not related to differences in saliva cortisol and plasma estrogen levels at the time of conception among the dietary groups in the present study. Especially PUFAs can positively affect the endocrine system, particularly the hypothalamicpituitary-adrenal and -gonadal axes and related cortisol, estrogen, and progesterone secretion rates, whereas EPA and AA are also precursors of prostaglandins. All these hormones interactively modulate reproductive processes, including the estrus cycle, conception, the process of gestation, and even parturition [3,39]. Although we found no differences in cortisol and estrogen concentrations, differences in the analyzed plasma fatty acids indicate modified availabilities of precursors for prostaglandin synthesis, cell membrane composition, and the energy balance.

The dietary supplementations significantly affected the plasma fatty acid status, determined by the percentage of single fatty acids, and, presumably, the availability of these molecules for metabolic functions. Supplementations with walnut oil (high in PUFAs) significantly decreased the percentage of SFAs and increased n-3 and n-6 PUFAs, resulting in the highest P:S ratios. Supplementations with coconut fat (high in SFAs) significantly increased the

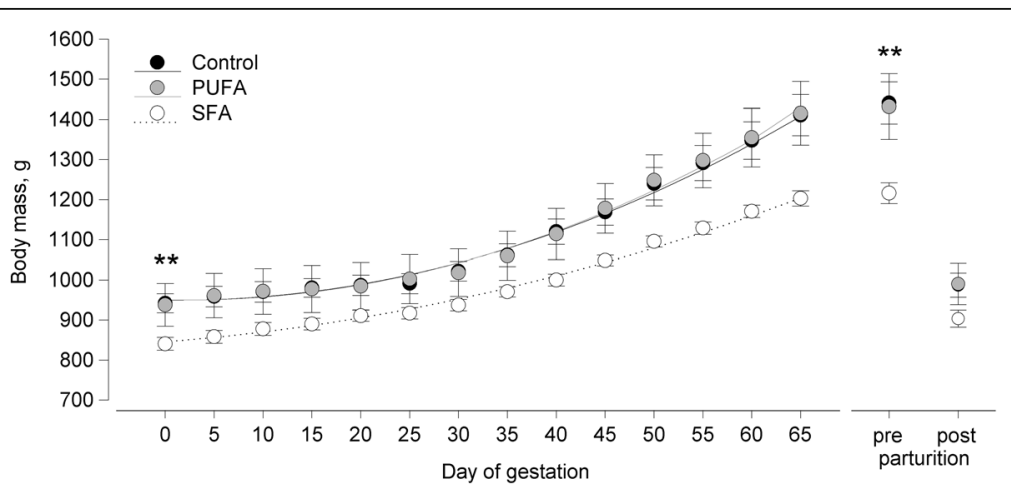

Fig. 1 Mean body mass in female guinea pigs maintained on a control, high-PUFA, or high-SFA diet during gestation and after parturition. Circles represent the body mass for each group and day (mean \pm s.e.m.); lines for gestation (day 0 to 65) represent the mean fitted values of a linear mixed effect model (corrected for repeated measurements) on the body mass change, including the second-order polynomial term for day and the total litter mass as covariate. Sample sizes: control $n=10$, PUFA $n=8$, SFA $n=10$. ${ }^{*} P \leq 0.01$ comparing SFA and the remaining groups. Group:day effect during gestation: $P \leq 0.05$ 


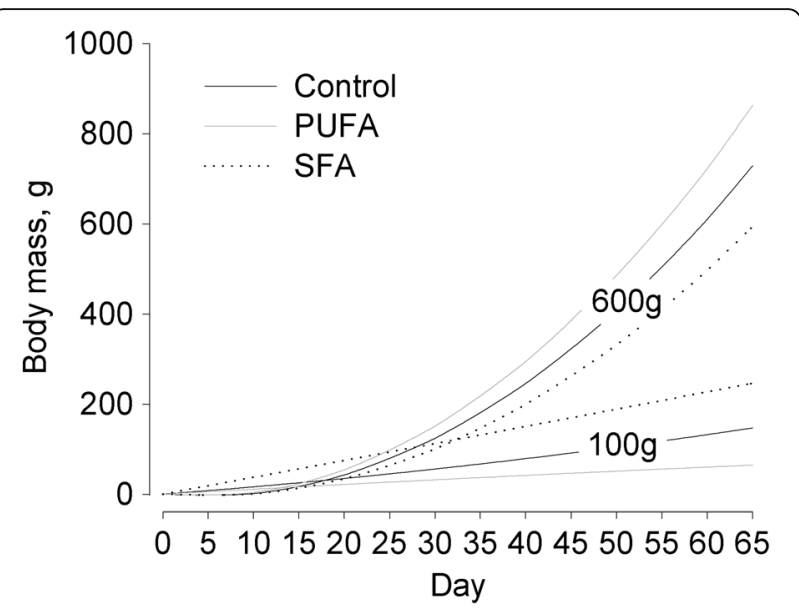

Fig. 2 Effect of total litter mass on the body mass gain during gestation in guinea pig females maintained on a control, high-PUFA, or high-SFA diet. Effects were extracted from a linear mixed effect model and are shown for a total litter mass of $100 \mathrm{~g}$ and $600 \mathrm{~g}$, respectively. Sample sizes: control $n=10$, PUFA $n=8$, SFA $n=10$. Group:day:total litter mass: $p \leq 0.05$

percentage of SFAs and decreased n-3 PUFAs, resulting in higher $\mathrm{n}-6: \mathrm{n}-3$ ratios in these animals. PUFAs and SFAs are seemingly metabolized in different ways: PUFAs are much faster oxidized and SFAs rather stored in abdominal fat $[11,40]$. A higher P:S ratio may therefore contribute to the short term energy supply, which would be required for maintaining daily physiological functions, while a lower $\mathrm{P}: \mathrm{S}$ ratio may indicate that more fat is stored. Hence, the significantly decreased body mass in SFA females at conception seems to be a contradiction, because a higher body fat accumulation could be assumed to result in a higher body mass. However, previous findings in guinea pigs indicate that a lower body mass can also be related to a higher percentage of body fat, although this was found in fetuses [41]. Energetic needs in SFA females were possibly not covered by direct energy supplies via dietary
PUFAs but rather via internal energy reserves and perhaps resulted in a reduced body mass. In relation to a lower $n-3$ PUFA status, this may have negatively influenced the reproductive output in SFA females, because especially $n-3$ PUFAs are suggested to promote reproductive functions [38]. However, as supplemented females did not exhibit a higher body mass at conception compared to control females, the ad libitum feeding of guinea pig pellets definitely counteracted a possible energetic imbalance among the dietary groups.

Dietary fatty acids differently affected the reproductive output in the studied animals and resulted in a relatively low litter size and total birth mass in the SFA group, which reduced the gestational effort for mothers in the following, as shown by the lowest body mass increase. The majority of SFA females gave birth to a maximum of three pups, whereas control and PUFA females had litter sizes of up to five pups. Ovulation rates and the number of produced and released ova may determine the litter size in rodents and can be highly affected by dietary PUFAs, with n-3 enhancing and n- 6 possibly decreasing the number of ova and pups per litter $[4,42]$. The increased plasma n-6:n-3 ratio in SFA females may have caused a lower ova production and decreased litter sizes. A possibly lower energy supply by dietary SFA intakes $[11,40]$ could have negatively affected the production of ova too, in contrast to higher P:S and lower $\mathrm{n}-6: \mathrm{n}-3$ ratios as found in PUFA females.

Unbalanced energy intakes in guinea pigs, including food restriction and overfeeding, can generally impact on reproduction and result in decreased litter sizes and individual birth mass $[41,43]$. Both diets high in PUFAs and SFAs can diminish these effects, resulting in an increased offspring body mass $[6,8]$. Interestingly, no differences were found in the individual birth mass among the dietary treatments in the present study, although birth mass tended to decrease with litter size.
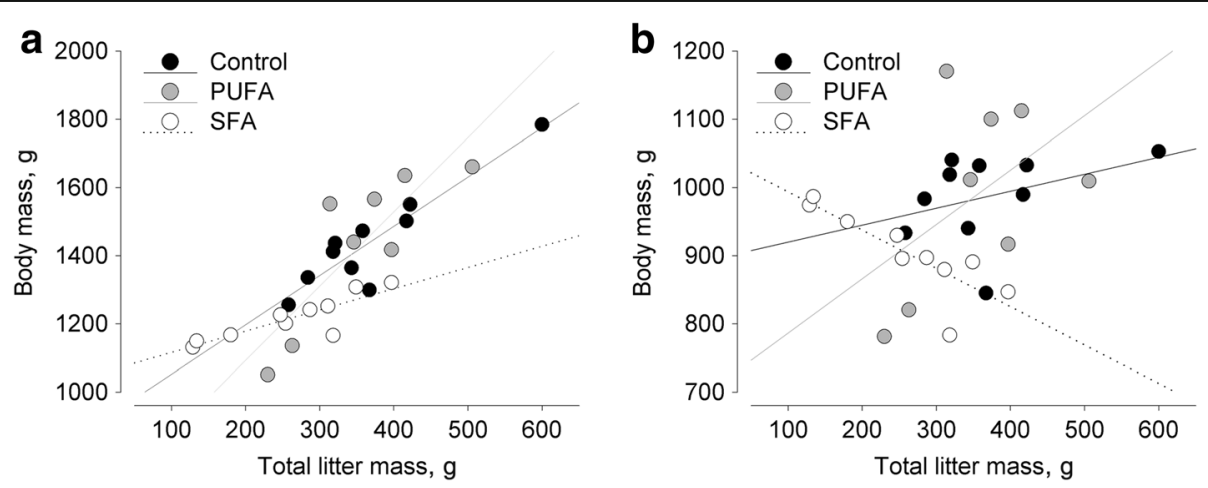

Fig. 3 Effects of total litter mass on body mass in guinea pig females maintained on a control, high-PUFA, or high-SFA diet. Effects are corrected for the body mass at conception. a Effects on body mass prior to parturition. Control: $p \leq 0.001$, PUFA: $p \leq 0.001$, SFA: $p \leq 0.001$; control vs. PUFA: n.s., control vs. SFA: n.s., PUFA vs. SFA: $p \leq 0.05$. b Effects on body mass after parturition. Control: n.s., PUFA: $p \leq 0.05$, SFA: n.s.; all group comparisons: n.s. (n.s.: not significant) 
Guinea pig pups born in larger litters usually show a lower body mass and also a higher mortality rate [13]. We did not detect such differences among the dietary groups, but a lower pup survival rate at birth was found in larger litters of control females. The fact that even dead-born pups were fully developed and still covered in their amniotic sacs indicates that they died at birth, probably because mothers did not rupture the amniotic sacs and the pups therefore asphyxiated soon after birth [44]. Mothers can be assumed to be exhausted by giving birth especially to large litters, whereas dietary fatty acids may provide additional energy during the process of parturition, thereby facilitating maternal care for the neonates, or even affect the morphology of the birth canal, resulting in an easier parturition. A higher energy intake and the resulting improved body condition of a mother may also favor the birth of male offspring [45], but we found no differences in the sex ratio at birth or in the ratio of dead and alive born male and female pups. These results are in contrast to a variety of mechanisms describing how diet and available energy may skew the sex ratio [46], which has also been reported for high-fat diets [9]. The present results suggest that guinea pigs generally produce more male offspring, which is apparently not affected by dietary fat intakes. As relatively low mortality rates and sex ratios higher than 0.5 at birth were detected in all dietary groups, it can be concluded that females used in this study were in good body conditions.

An increased survival rate in large litters is not only of major importance regarding the lifetime reproductive success, but also in terms of a mother's investment during gestation, as reflected in the relative body mass increase during gestation caused by the developing fetuses. A very high body mass increase during gestation represents a significant energetic effort in guinea pigs especially during the second half of gestation [47]. As the litter size and total birth mass in SFA mothers were generally lower, at least compared to PUFA females, this obviously resulted in a reduced gestational effort in these animals. PUFA females, in contrast, showed highest gestational efforts and were seemingly able to support the developing pups especially in case of larger litters by an increased energetic investment during gestation, which resulted in a similar individual birth mass among different litter sizes and groups. An increased energy allocation in the developing fetuses could have also eliminated a negative relationship between litter size and individual birth mass, which usually occurs in guinea pigs [13, 14]. The relatively low litter sizes and total litter masses in SFA females, which resulted in the low gestational efforts, perhaps enabled these animals to provide the developing fetuses with adequate energy, which might have not been possible in case of larger litters.
All females revealed a significantly increased body mass after parturition compared to conception, indicating available internal energy reserves for the lactation period after the exhausting parturition. Although female guinea pigs energetically invest more in their offspring during gestation compared to lactation [16], the short lactation period is usually characterized by a regular decrease in the mothers' body mass [26]. SFA females in the present study showed the most pronounced body mass gain from conception to post parturition. This may indicate that these animals were able to accumulate higher internal energy reserves during gestation, perhaps due to the lower number of developing fetuses and a related lower energetic investment. PUFA females, however, seemed to adjust their own body mass to the litter size and total litter mass, as the total litter mass positively affected the mother's body mass after parturition. This was not detected in control and SFA females and also a previous study in guinea pigs did not reveal such an effect [43]. However, a higher gestational effort, litter size, and total litter mass could be assumed to impair a mother's body condition after parturition. As this is apparently not the case in guinea pigs, it can be concluded that these animals are able to accumulate additional energy reserves during gestation, which may be enhanced by feeding on dietary PUFAs. A lower litter size and total litter mass in SFA females may have further been related to less pronounced placental tissues [48], which perhaps also caused a decreased energy allocation in establishing and maintaining these tissues. This would also be supported by a general positive link to the total litter mass, as more and heavier fetuses require more space in the uterus and have to be provided with more oxygen and nutrients, resulting in larger and heavier placentas [41].

\section{Conclusions}

SFAs seem to reduce the litter size in guinea pigs, which would reduce the lifetime reproductive success probably due to inadequate available energy for reproductive functions and for developing a higher number of fetuses. In this way, SFAs may also decrease the gestational effort, which perhaps favors body condition in these animals during and after gestation due to a lower energy allocation required for the developing fetuses. Dietary PUFAs seem to modify energy accumulations in pregnant guinea pigs in relation to the litter size and total litter mass towards the maximum reproductive success. Although both types of fatty acids seem to affect female reproduction at different levels, they may both maximize the survival rate at birth and therefore the different gestational efforts would be justified. These promising results may further help to understand how dietary fatty acids can modulate reproductive processes and the reproductive success, perhaps by primarily affecting the 
mother's body condition. This demonstrates the significant influence of dietary fatty acids on mammalian reproduction, since not only the developing fetuses but also a mother's reproductive performance may be highly influenced by an adjustment of pre-fertilization dietary PUFA and SFA intakes. However, a limitation of this study is the lack of knowledge which male fertilized the female, because a high rate of multiple paternities could have been possible due to our experimental setup [49]. Paternal effects in relation to dietary fatty acids (e.g. number of mutation rates [50]) should be considered in order to fully interpret the whole impact of these nutrients on reproduction in mammals.

\section{Additional file}

Additional file 1: Dataset.xls; Dataset supporting the results and

conclusions of this article. (XLS 56 kb)

\section{Abbreviations}

AA: Arachidonic acid; AIC: Akaike information criterion; ALA: a-linolenic acid ANOVA: Analysis of variance; DHA: Docosahexaenoic acid; EPA: Eicospentaenoic acid; FAMES: Fatty acid methyl esters; LA: Linoleic acid; LME: Linear mixed effects model; n-3: Omega-3; n-6: Omega-6; PUFA: Polyunsaturated fatty acid; SFA: Saturated fatty acid

\section{Acknowledgements}

The authors would like to thank the following staff of the University of Vienna: Alexandra Christian and Verena Brudermann, for the help in animal keeping, and Elisabeth Pschernig, responsible for hormonal analyses. We would also like to thank Michael Nemeth for his help in constructing the experimental setup.

\section{Funding}

MN was supported by a PhD completion grant of the University of Vienna during the preparation of the manuscript. The funders played no role in study design, performance of the experiment, data analysis, or writing of the manuscript.

\section{Availability of data and materials}

All data generated or analyzed during this study are included in this published article and its Additional file 1.

\section{Authors' contributions}

MN designed and performed the experiment, analyzed the data, and wrote the manuscript. EM designed the experiment and edited the manuscript. CS performed the experiment, analyzed the data and edited the manuscript. KHW performed the experiment and edited the manuscript. RQ performed the experiment and edited the manuscript. BW designed the experiment, wrote and edited the manuscript. All authors read and approved the final manuscript.

\section{Competing interests}

The authors declare that they have no competing interests.

\section{Consent for publication}

Not applicable.

\section{Ethics approval and consent to participate}

Experiments were conducted in accordance with EU Directive 2010/63/EU for animal experiments and the Austrian laws for animal experiments and animal keeping. The study has been checked and approved by the internal board on animal ethics and experimentation of the Faculty of Life Sciences of the University of Vienna (\# 2014-005) and the Austrian Federal Ministry of Science and Research (BMWF-66.006/0024-II/3b/2013).

\section{Author details}

'Department of Behavioural Biology, University of Vienna, Althanstrasse 14 1090 Vienna, Austria. ${ }^{2}$ Department of Nutritional Sciences, University of Vienna, Althanstrasse 14, 1090 Vienna, Austria. ${ }^{3}$ Department of Anthropology, University of Vienna, Althanstrasse 14, 1090 Vienna, Austria.

Received: 2 September 2016 Accepted: 14 March 2017

Published online: 01 April 2017

\section{References}

1. Schneider JE. Energy balance and reproduction. Physiol Behav. 2004;81:289-317.

2. Funston RN. Fat supplementation and reproduction in beef females. J Anim Sci. 2004;82(E-Suppl):E154-61.

3. Wathes DC, Abayasekara DRE, Aitken RJ. Polyunsaturated fatty acids in male and female reproduction. Biol Reprod. 2007;77:190-201.

4. Broughton KS, Bayes J, Culver B. High a-linolenic acid and fish oil ingestion promotes ovulation to the same extent in rats. Nutr Res. 2010;30:731-8.

5. Ambrose DJ, Kastelic JP, Corbett R, Pitney PA, Petit HV, Small JA, et al. Lower pregnancy losses in lactating dairy cows fed a diet enriched in a-linolenic acid. J Dairy Sci. 2006:89:3066-74.

6. Rebollar PG, García-García RM, Arias-Álvarez M, Millán P, Rey Al, Rodríguez $M$, et al. Reproductive long-term effects, endocrine response and fatty acid profile of rabbit does fed diets supplemented with n-3 fatty acids. Anim Reprod Sci. 2014;146:202-9.

7. Santillán ME, Vincenti LM, Martini AC, Fiol de Cuneo M, Ruiz RD, Mangeaud A, et al. Developmental and neurobehavioral effects of perinatal exposure to diets with different $\omega-6: \omega-3$ ratios in mice. Nutrition. 2010;26:423-31.

8. Bilbo SD, Tsang V. Enduring consequences of maternal obesity for brain inflammation and behavior of offspring. FASEB J. 2010;24:2104-15.

9. Alexenko AP, Mao J, Ellersieck MR, Davis AM, Whyte JJ, Rosenfeld CS, et al. The contrasting effects of Ad libitum and restricted feeding of a diet very high in saturated fats on sex ratio and metabolic hormones in mice. Biol Reprod. 2007;77:599-604

10. Fernandez ML, West KL. Mechanisms by which dietary fatty acids modulate plasma lipids. J Nutr. 2005;135:2075-8.

11. Sanz M, Lopez-Bote CJ, Menoyo D, Bautista JM. Abdominal fat deposition and fatty acid synthesis are lower and $\beta$-oxidation is higher in broiler chickens fed diets containing unsaturated rather than saturated fat. J Nutr. 2000;130:3034-7.

12. Künkele J. Effects of litter size on the energetics of reproduction in a highly precocial rodent the guinea pig. J Mammal. 2000;81:691-700

13. Manjeli Y, Tchoumboue J, Njwe RM, Teguia A. Guinea-pig productivity under traditional management. Trop Anim Health Prod. 1998;30:115-22.

14. Czarnecki $R$, Adamski M. Factors influencing litter size and birthweight in the newborn long-haired Guinea pigs (Cavia aperea f. porcellus). J Appl Anim Res. 2016:44:71-6.

15. Künkele J, Trillmich F. Are precocial young cheaper? Lactation energetics in the guinea pig. Physiol Zool. 1997;70:589-96.

16. Künkele J. Energetics of gestation relative to lactation in a precocial rodent, the guinea pig (Cavia porcellus). J Zool. 2000;250:533-9.

17. Berry M, Gray C, Wright K, Dyson R, Wright I. Premature guinea pigs: A new paradigm to investigate the late-effects of preterm birth. J Dev Orig Health Dis. 2015;6:143-8

18. ssniff ${ }^{\circledast}$ Complete feeds for rabbits and guinea pigs. http://www.ssniff.com/ documents/04_catalogue_rabbit_guinea_engl_1.pdf. Accessed 7 Dec 2016.

19. Ferraz AC, Delattre AM, Almendra RG, Sonagli M, Borges C, Araujo P, et al. Chronic $\omega$-3 fatty acids supplementation promotes beneficial effects on anxiety, cognitive and depressive-like behaviors in rats subjected to a restraint stress protocol. Behav Brain Res. 2011;219:116-22.

20. Nemeth M, Millesi E, Wagner KH, Wallner B. Sex-Specific Effects of Diets High in Unsaturated Fatty Acids on Spatial Learning and Memory in Guinea Pigs. PLoS One. 2015;10:e0140485.

21. R Core Team. R: A language and environment for statistical computing. Vienna: R Foundation for Statistical Computing; 2015. http://www.R-project.org/.

22. Nemeth M, Millesi $E$, Wagner $\mathrm{KH}$, Wallner B. Effects of diets high in unsaturated fatty acids on socially induced stress responses in guinea pigs. PLoS One. 2014;9:e116292.

23. Spector AA. Plasma free fatty acid and lipoproteins as sources of polyunsaturated fatty acid for the brain. J Mol Neurosci. 2001;16:159-65.

24. Sachser N, Dürschlag M, Hirzel D. Social relationships and the management of stress. Psychoneuroendocrinology. 1998;23:891-904. 
25. Weir BJ. The management and breeding of some more hystricomorph rodents. Lab Anim. 1970;4:83-97.

26. Schöpper H, Palme R, Ruf T, Huber S. Chronic stress in pregnant guinea pigs (Cavia aperea f. porcellus) attenuates long-term stress hormone levels and body weight gain, but not reproductive output. J Comp Physiol B. 2011;181: 1089-100.

27. Ishii O. Observations on the sexual cycle of the guinea pig. Biol Bull. 1920; 38:237-50.

28. Nemeth M, Pschernig E, Wallner B, Millesi E. Non-invasive cortisol measurements as indicators of physiological stress responses in guinea pigs. Peer J. 2016:4:e1590.

29. Palme R, Möstl E. Biotin-streptavidin enzyme immunoassay for the determination of oestrogens and androgens in boar faeces. In: Görög S, editor. Proceedings of the 5th Symposium on the Analysis of Steroids. Budapest: Akademiai Kiado; 1993. p. 111-7.

30. Palme R, Möstl E. Measurement of cortisol metabolites in faeces of sheep as a parameter of cortisol concentration in blood. Z Säugetierkd. 1997;62:192-7.

31. Wagner KH, Auer E, Elmadfa I. Content of trans fatty acids in margarines, plant oils, fried products and chocolate spreads in Austria. Eur Food Res Technol. 2000;210:237-41.

32. Pinheiro J, Bates D, DebRoy S, Sarkar D, R Core Team. nlme: Linear and Nonlinear Mixed Effects Models. R package version 31-122. 2015. http:// CRAN.R-project.org/package=nlme.

33. De Rosario-Martinez H. phia: Post-Hoc Interaction Analysis. R package version 0.2-1. 2015. http://CRAN.R-project.org/package=phia.

34. Pohlert T. The Pairwise Multiple Comparison of Mean Ranks Package (PMCMR). R package. 2014. http://CRAN.R-project.org/package=PMCMR.

35. Fox J. Effect Displays in R for Generalised Linear Models. J Stat Softw. 2003;8:1-27.

36. Sullivan EL, Riper KM, Lockard R, Valleau JC. Maternal high-fat diet programming of the neuroendocrine system and behavior. Horm Behav. 2015;76:153-61.

37. Innis SM. Perinatal biochemistry and physiology of long-chain polyunsaturated fatty acids. J Pediatr. 2003;143:S1-8.

38. Gulliver CE, Friend MA, King BJ, Clayton EH. The role of omega-3 polyunsaturated fatty acids in reproduction of sheep and cattle. Anim Reprod Sci. 2012;131:9-22.

39. Abayasekara DRE, Wathes DC. Effects of altering dietary fatty acid composition on prostaglandin synthesis and fertility. Prostaglandins Leukot Essent Fatty Acids. 1999;61:275-87.

40. Shimomura $Y$, Tamura T, Suzuki M. Less body fat accumulation in rats fed a safflower oil diet than in rats fed a beef tallow diet. J Nutr. 1990:120:1291-6.

41. Kind KL, Roberts $C T$, Sohlstrom Al, Katsman A, Clifton PM, Robinson JS, et al. Chronic maternal feed restriction impairs growth but increases adiposity of the fetal guinea pig. Am J Physiol Regul Integr Comp Physiol. 2005;288: R119-26.

42. Trujillo EP, Broughton KS. Ingestion of n-3 polyunsaturated fatty acids and ovulation in rats. J Reprod Fertil. 1995;105:197-203.

43. Michel $\mathrm{CL}$, Bonnet $\mathrm{X}$. Influence of body condition on reproductive output in the guinea pig. J Exp Zool. 2012;317:24-31.

44. Tejml P, Šoch M, Brouček J, Jirotková D, Smutný L, Zábranský L, et al. Factors influencing behaviour of guinea pig females during the birth. Adv Environ Sci Energy Plann. 2015;2015:81-4.

45. Trivers RL, Willard DE. Natural selection of parental ability to vary the sex ratio of offspring. Science. 1973;179:90-2.

46. Rosenfeld CS, Roberts RM. Maternal diet and other factors affecting offspring sex ratio: A review. Biol Reprod. 2004;71:1063-70.

47. Engle WA, Lemons JA. Composition of the fetal and maternal guinea pig throughout gestation. Pediatr Res. 1986;20:1156-60.

48. Elias AA, Ghaly A, Matushewski B, Regnault TRH, Richardson BS. Maternal Nutrient Restriction in Guinea Pigs as an Animal Model for Inducing Fetal Growth Restriction. Reprod Sci. 2016;23:219-27.

49. Asher M, Lippmann T, Epplen JT, Kraus C, Trillmich F, Sachser N. Large males dominate: ecology, social organization, and mating system of wild cavies, the ancestors of the guinea pig. Behav Ecol Sociobiol. 2008;62:1509-21.

50. Kong A, Frigge ML, Masson G, Besenbacher S, Sulem P, Magnusson G, et al. Rate of de novo mutations, father's age, and disease risk. Nature. 2012;488:471-5

\section{Submit your next manuscript to BioMed Central and we will help you at every step:}

- We accept pre-submission inquiries

- Our selector tool helps you to find the most relevant journal

- We provide round the clock customer support

- Convenient online submission

- Thorough peer review

- Inclusion in PubMed and all major indexing services

- Maximum visibility for your research

Submit your manuscript at www.biomedcentral.com/submit
Biomed Central 